\title{
POLLEN- AND SEED-STERILITY IN HYBRIDS 1
}

\section{BRADLEy MoORE Davis}

Sterility of hybrids in various forms and degrees is a phenomenon so frequently presented to the plant breeder and geneticist that in some form and in some degree it is rather to be expected. The first problem in its study demands a critical examination to determine in the life history the place of those conditions that bring about the sterility in question. In earlier days the gametes were generally expected to carry the blame of failure to reproduce the line. More recent studies have shown that responsibilities for sterility cannot be so easily placed.

Sterility, as expressed by varying proportions of abortive pollen and abortive ovules, is very common, and since it is easily recognized this manifestation of sterility has received the greatest share of attention. It is expressed by failure of the pollen grain to attain full size, the structure shriveling and usually losing its protoplasmic contents. In a like manner, the megaspore or embryo sac does not reach a normal development in the ovule. Such behavior results in failure to produce gametes, and cytological studies, as far as they have gone, indicate that this form of sterility, at least frequently, has its causation in irregularities of the reduction divisions which immediately precede the differentiation of micro- and megaspores.

During these mitoses spindles may not be normal in form, chromosomes may be distributed in varying and irregular numbers, and the preparations for the reduction divisions may show abnormalities. Such phenomena clearly indicate a breakdown in the mechanism of nuclear division at this critical stage in the life history. It seems reasonable to assume in these cases that the hybrid must carry a germ plasm the structural elements of which can not conduct themselves in the orderly manner so characteristic of meiosis. Speculation on the reasons for the obvious breakdown of the cell and nuclear mechanism at this point in the life history would lead us too far afield for the purposes of this paper. It seems clear, however, that the causes lie in the heterozygous nature of the germ plasm, since we do not find this form of sterility in pure material. Abnormalities of chromosome distribution are clearly invited when the two sets are of different genetical constitution, since irregularities of segregation are rendered much more likely.

It should not be assumed, however, that the presence of abortive pollen and abortive ovules is proof positive that the parent plant is hybrid, although

${ }^{1}$ Read in the symposium on "Sterility in Plants," at the joint meeting of Section G of the American Association for the Advancement of Science, the Botanical Society of America, and the American Phytopathological Society, at Cambridge, December 27, 1922. 
any plant showing high proportions of shriveled pollen may very justly be an object of suspicion. There is a form of pollen sterility due to malnutrition, and this condition may be brought on experimentally by operations seriously affecting the vegetative activities of a plant, as, for example, stripping the leaves from stems. In this connection should be mentioned a form of abortion presented in heterosporous plants when one or more megaspores, perhaps through more favorable position or the good fortune of a better start, are able to develop at the expense of their neighbors which, giving up their substance, are sacrificed for the good of those megaspores that survive. This is fundamentally a form of sterility due to malnutrition, but it is not of course peculiar to hybrids.

Sterility which results from failure to develop gametes is one thing, and sterility due to the inability of gametes to unite is quite another. The latter form of sterility is much more difficult of study in plant material than in animal because the fusion of gametes in higher plants takes place in structures and tissues difficult of examination. Zoölogists recognize the phenomenon and it may be expected to be present in plants, but forms most profitable for study seem rather more likely to be found in groups of the thallophytes than among the higher plants. Sterility of this character need not be due to particular physical or chemical conditions that prevent the union of gamete protoplasts. Pollen grains may not find the secretions of the stigma favorable for their germination. There is probably a very large amount of sterility which results from the inability or slowness of some pollen tubes to penetrate certain lengths of style. We know something of this matter through the studies of East on Nicotiana, of D. F. Jones with pollen mixtures in maize, and from observations of Buchholz and Blakeslee on pollen-tube growth in Datura. It is probably a factor of importance in determining some of the results of Oenothera breeding.

Zygotic sterility is very common among plant hybrids. It means that the zygote is either unable to develop at all or that it produces an embryo which dies early in the production of a seed-like structure. In either case this form of sterility may be suspected from the presence of shriveled ovules or shriveled seed-like structures of various sizes, but generally smaller than normal seeds. Sometimes there may be structures as large as seeds and externally like them but without embryos. Zygotic sterility is therefore conveniently recognized by seed sterility, only care must be taken to make sure that seeds are really sterile because of internal conditions and not merely slow of germination. In plant genetics it is necessary to know these facts before conclusions can be drawn on the significance of so-called "percentages of germination." Conditions must be arranged to force seeds to complete germination, and examinations of the residue of ungerminated seed-like structures must be made to make sure that germination is really complete.

Of course it is impossible to draw a fast line between the embryo which dies in the seed and the embryo which comes forth a weakling unable to 
live long as a seedling. The geneticist working in certain groups knows this latter type of seedling very well. Oenothera material is full of illustrations. One type is that of a seedling which expands green cotyledons but the hypocotyl is totally unable to develop a root. Other forms are delicate, frequently etiolated seedlings which live only a few days even when nursed along with most particular attention to their needs of suitable soil, careful watering, and cooler temperatures. Perhaps some of these seedlings are accidents of development, but it is clear from many studies that there are large groups in which the inability to develop rests on a genetical basis and is really the expression of a degree and form of sterility.

Thus, from forms of zygotic sterility expressed by abortive embryos in seed-like structures, we pass insensibly to conditions illustrated by classes of weak seedlings which make a start only to die sooner or later. This is truly a form of sterility when the behavior is due to the germinal constitution of the seedling, and it is impossible to draw lines sharply in the wide range expressed by the various degrees of impotence. Examples of this form of sterility are well known to every plant geneticist who follows carefully the fate of seedlings from hybrid material when germination is complete. It not infrequently happens that large groups of etiolated seedlings appear, or weak dwarfs of various forms, or plants which make considerable growth but fail to mature. Most of these products die early even with the best of care; very few will live under the conditions of the open garden.

In the foregoing sketch $I$ have endeavored to make clear the fact that sterility in plants is expressed in a number of very different forms and may operate at several points in the life history. The fact that the life history of plants is made up of two generations, gametophyte and sporophyte, with the three critical periods of chromosome segregation, fertilization, and embryo development frequently associated with involved physiological and histological conditions adds greatly to the complications of observation and interpretation and makes the study of sterility in plants more difficult than that of animals.

With so many forms of sterility known and expressed in such various ways, the specific causes must be very numerous. Some of them may be relatively simple, as when pollen fails to function because of delay in germination or slowness of tube growth. Even a breakdown at the time of the reduction divisions may be something of an accident when due to chance irregularities of chromosome distribution. On the other hand, sterility based on inability of two sorts of germ plasm to work in harmony, whether in the beginning or at the end of a life history, presents problems that are difficult to vision.

The geneticist postulates, as a form of expression, lethal factors in expressing results of his experimental work when sterility appears, and his formulae are of course justified as steps towards an understanding of finalities. The lethals of the geneticist are placed as genes in the chromo- 
somes, and it must be confessed that we cannot show any reasons why they may not be there even though their presence adds mightily to the responsibilities of these heavily worked cell structures. We cannot say that slowness of germination on the part of a pollen grain may not be due to the character of substance or lack of substance in some region of a chromosome even though the pollen tube grows through activity of the ectoplast. On the other hand it may be that some lethals are present outside of nuclear structures. Breeding studies should be able to separate cytoplasmic lethals, if such exist, from those that are associated with chromosomes, since the two would be expected to follow different systems in inheritance.

We have treated sterility as though it depended in the last analysis on factors internal to the organism which may be postulated as genes and which express themselves through inheritance. With respect to sterility in hybrids, its association with germ-plasm organization is generally evident. It is a fair question to ask whether sterility ever has a basis other than that of germ-plasm organization. The temptation is sometimes great to lay the responsibilities of sterility to causes outside the organism and thus to separate a sort of physiological sterility from that which has a genetical relation. There are, for example, forms of sterility brought out by disease or by physiological conditions harmful to the plant. Supposing such sterility to be only partial, as is frequently the case, we should not of course expect the inheritance of this acquired sterility for the same reasons that we do not expect the inheritance of an acquired morphological character. So far as I am aware there are no reasons to regard sterility as other than a characteristic dependent upon germ-plasm organization and dependent in the same sense as are morphological characters.

As stated at the beginning of this paper, hybrid material is generally expected to exhibit in some degree sterility of various sorts. Hybrid material is also generally expected to show its heterozygous nature in breeding by throwing a varied assortment of forms in its progeny. There is coming to be recognized, however, a type of hybrid that reproduces itself perfectly, throwing at most only occasional variants. Such hybrids satisfy fully our concept of a species as a kind of animal or plant which breeds true. They are impure species because their germ plasm in the diploid condition carries different sets of genes affecting characters other than those of sex. The pure species in contrast has a germ plasm carrying two similar sets of genes each contributed by one of the parents and each with the same genetical constitution except when genes responsible for sex and sex-linked characters are concerned.

Because the impure or hybrid species is of particular interest in relation to the problems of hybrid sterility I must discuss certain phases of this subject although they have been treated with some fullness in my earlier paper "Species, pure and impure." 2

${ }^{2}$ Davis, B. M. Species, pure and impure. Sci., n. ser. 55: I08-II4. 1922. 
The impure species, although hybrid in its germinal constitution, breeds true because only. such gametes unite and give progeny as will reproduce the heterozygdus constitution of the parent plant. Other types of gametes to be expected from the segregation divisions of meiosis either do not develop or fail to function for one of various possible reasons, or, if they do unite, the zygote either can not develop at all or it produces an embryo or seedling which can not mature. In short, there are breakdowns at one or more of various critical points in the life history, and thus some form of sterility eliminates the development of all or nearly all groups of segregates possible to the hybrid in question, and only such combinations of gametes are effective as will give the genotype of the parent.

This concept of the pure-breeding hybrid is not a fancy. We have excellent evidence that impure species are common in the genus Oenothera and that Oenothera Lamarckiana is one of them. Certain lines of Drosophila are known to be impure, and we owe to studies of Muller on such material the theory of balanced lethals which offers the best notion of a mechanism in heredity responsible for the generally true breeding of an impure species and for the appearance of occasional variants which some geneticists call mutarlts but which are really segregates from the heterozygous stock.

The theory of balanced lethals postulates the presence of two different lethals, for example $x$ and $y$, the first in one chromosome and the second in the other chromosome of a synaptic pair. The organism is therefore heterozygous for each lethal. The theory also assumes that each lethal is effective only in double dose. The reduction divisions in such material will give two classes of sperms and two classes of eggs, each class distinguished by the presence of one of the two lethals. Thus there will be sperms $x$ and $y$ and eggs $x$ and $y$ and the chance mating of these will give zygotes in the following proportions $\mathrm{I} x x: 2 x y: \mathrm{I} y y$. Zygotes $x x$ and $y y$, because they have lethals in double dose cannot develop progeny, but the zygotes with the heterozygous combination $x y$ will live and reproduce the impure or hybrid parent type. Thus an impure species or race will breed true and maintain a constant state of hybridism unless the relative position of the lethals is changed by a crossover or unless a lethal becomes ineffective through a mutation. A crossover makes possible a class of zygotes free from both lethals, because sperms and eggs would be of the two classes $x y$ and $o o$ and the zygotes would be in the proportions Ixxyy:2xy:10o. Through the class of segregates free from both lethals recessive characters would appear if genes responsible for their suppression were removed by way of the class homozygous for both lethals. The appearance of such recessives will simulate mutations although in reality they are manifestations of a process of segregation.

There is not time to carry farther a discussion of the bearing of the theory of lethals on the facts of hybrid sterility. It is not probable that conditions in Oenothera and Drosophila, two groups which have received 
exceptional attention, are marked exceptions among animals and plants. Intensive studies on other forms are more than likely greatly to extend our recognition of the presence in nature of impure lines and impure species. Hybridization in itself probably invites the development of lethals in proportion as the mixing of diverse germ plasms disturbs delicate and vital adjustments and creates confusion in orderly processes of development.

The subject of lethals and impure species has come to have particular interest for the student of certain groups of plants which are conspicuous components of floras. The systematics of Oenothera has reached a stage so complex that much material can not be identified in the field and species may be determined only when their behavior is studied in the experimental garden. Systematic studies on violets and brambles have employed similar methods of genetical analysis, and many other groups will require the same sort of treatment. Then there are those large assemblages characterized by high degrees of self-sterility, conditions probably not uncommon in the Compositae. Here the progeny is always or usually crossbred as in all unisexual animals and plants. Again, even when self-fertilization is possible, it has been found in some material that inbred lines are not so vigorous as the outbred and thus conditions favor hybridity.

We open a paper with a discussion of pollen- and seed-sterility in hybrids. We are led at the end to touch upon some of the most complex problems of genetics and taxonomy.

UNIVERSITY OF MichigaN 